\title{
Energy dissipation in atomic-scale friction
}

\author{
Yuan-zhong HU ， Tian-bao MA, Hui WANG \\ State Key Laboratory of Tribology, Tsinghua University, Beijing 100084, China \\ Received: 09 November 2012 / Revised: 25 January 2013 / Accepted: 01 February 2013 \\ (C) The author(s) 2013. This article is published with open access at Springerlink.com
}

\begin{abstract}
The mechanisms of energy dissipation are discussed in this paper by reviewing the models and research in atomic-scale friction. The study is undertaken to answer a fundamental question in the study of friction: How is frictional work dissipated, particularly in cases where material damage and wear are not involved. The initiation of energy dissipation, the role of structural commensurability, and the estimation of the interfacial shear strength have been examined in detail by introducing the Tomlinson model, the FrenkelKontorova model, and the cobblestone model, respectively. The discussion is extended to energy dissipation progress described in terms of phononic and electronic damping. The contributions from other mechanisms of dissipation such as viscoelastic relaxation and material wear are also included. As an example, we analyzed a specific process of dissipation in multilayer graphene, on the basis of results of molecular dynamics (MD) simulations, which reveal a reversible part of energy that circulates between the system and the external driver. This leads us to emphasize that it is crucial in future studies to clearly define the coefficient of dissipation.
\end{abstract}

Keywords: energy dissipation; atomic-scale friction; phononic damping; viscoelastic relaxation

\section{Introduction}

Why does friction exist universally between surfaces in relative motion? How is it created in the first place? These are fundamental questions that Leonardo da Vinci considered more than 500 years ago, and the questions that scientists and engineers have asked over the centuries. Researchers such as G. Amontons, C. A. Coulomb, F. P. Bowden, I. V. Kragelskii, and D. Tabor have tried to provide answers to these questions, but satisfactory explanation has not been presented thus far.

In early studies, friction was assumed to result from engaged surface asperities or from interacting surface molecules [1]. As an explanation for the origin of friction, however, both models are unsatisfactory in the sense that they are unable to describe precisely how the engagement of asperities or interaction of molecules lead to energy dissipation in the process of

* Corresponding author: Yuan-zhong HU.

E-mail: huyz@tsinghua.edu.cn friction. In the case of sliding, friction used to be regarded as the force acting in the opposite direction of motion, and a certain amount of work would need to be done by the force to sustain the sliding motion. Frictional work is eventually converted into heat, suggesting that friction is in fact a process of energy transformation. This makes energy dissipation a crucial scientific issue in understanding friction.

Historically, energy dissipation was not considered a problem difficult to explain as long as the frictional work was assumed to be dissipated through plastic deformation and material damage [2]. This is true for clean metal surfaces in contact and sliding. However, if the surfaces are contaminated or oxidized, or if surface films/lubricants are artificially applied as in boundary lubrication, very little plastic deformation occurs and the friction is then mainly attributed to interfacial effects. Similar effects also appear in the sliding of materials like polymer and ceramics. Friction without plastic deformation and wear raises many problems, still unsolved, concerning the nature of energy dissipation. 
The problem of explaining energy dissipation has generally been recognized by scientists through the end of the $20^{\text {th }}$ century [3], but it was David Tabor who clearly identified the problem and brought it to the attention of the tribology community twenty years ago in a NATO-sponsored conference on the Fundamentals of Friction. In a lecture presented at the conference, he suggested a mechanism of energy dissipation for the friction in elastic or near elastic sliding, which can be summarized as follows. (i) Atoms at the interface are displaced from their equilibrium position. (ii) The displaced atoms reach an unstable configuration, and at this point they flick back to a new equilibrium position. (iii) The strain energy is lost in the form of atomic vibrations and these in turn are degraded into heat [4]. As pointed out by Tabor himself, the basic ideas listed above can be found in a paper by Tomlinson [5], who recognized the role of mechanical instability in frictional energy dissipation, but for some reason did not emphasize the important role of atomic vibrations.

There has been an increase in research on friction in the years following the NATO conference. This is accompanied by rapid development of molecular dynamics (MD) simulations and successful applications of new scientific instruments, such as atomic force microscope (AFM), surface force apparatus (SFA), and quartz crystal microbalance (QCM). The research has led to the emergence of new terms in the tribology community, e.g., interfacial friction to emphasize the friction that occurs only at the contact interface, wearless friction to distinguish the friction without wear from the wear-induced process, and atomic-scale friction to identify the dimensions of the system and contributions from atomic interactions.

In this article, we review recent progress in studies of interfacial or atomic-scale friction, including models, experiments, and simulations, but the focus is on the theoretical developments in this field, in particular, on the discussions pertaining to energy dissipation. The discussions have been limited to kinetic friction when two solid surfaces slide past each other. The issue of frictional dissipation by purely viscous effect is not included in this paper, but Klein and coworkers $[6,7]$ have published remarkable results on this issue, which reveal the mechanism of lubrication provided by hydration layers and polymer brushes with very low friction. For more details, readers are referred to the review article of "Hydration lubrication" by Klein in the current issue of "Friction" [8].

This report is arranged in the following manner. The next three sections are dedicated to descriptions of three models for atomic-scale friction, the Tomlinson model, the Frenkel-Kontorova (FK) model, and the cobblestone model. Section 5 examines the progress of dissipation in terms of phononic and electronic damping. Contributions from other dissipation mechanisms such as viscoelastic relaxation and material wear are discussed in Section 6. Section 7 describes a specific process of energy dissipation based on MD simulations for multilayer graphene, which reveals a reversible component of dissipation energy. Finally, concluding remarks are given in Section 8.

\section{Initiation of energy dissipation - the Tomlinson model}

The idea that mechanical instability of an atomic system would result in energy dissipation was attributed to a paper by Tomlinson in 1929, but it was discovered recently that a similar idea was published independently by Ludwig Prandtl in German, one year before Tomlinson [9]. It has been suggested that the model be renamed as the Prandtl-Tomlinson model. In this paper, however, we still use the traditional term Tomlinson model for brevity. In spite of the idea being proposed a long time ago, it was more than 50 years later when McClelland [3] presented a clear description of the model and a comprehensive analysis of the dynamics of the system in which a row of independent oscillators slide over a periodic potential, as shown in Fig. 1. The oscillators, representing interfacial atoms, do not interact with each other but each of them is connected by a single spring to a rigid support $B$, and the periodic potential describes the interactions from the opposite solid A.

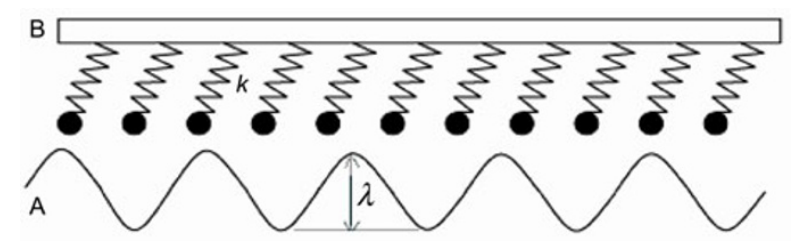

Fig. 1 Schematic of the Tomlinson model. 
Dynamic analysis for the system results in a criterion of stability that if support B is slid infinitesimally slowly relative to solid $A$, the sliding will become unstable under the following condition,

$$
k \leqslant-\frac{\partial^{2} V}{\partial x^{2}}
$$

where $k$ is the spring stiffness, $V$ is the potential function, and $x$ denotes the oscillator position in the sliding direction. The essence of the Tomlinson model is to clarify that energy dissipation initiates from mechanical instability, i.e., the energy is dissipated by the instability induced stick-slip motion and consequent atomic vibrations. One should keep in mind that the Tomlinson stick-slip is different from the usual macroscopic stick-slip, and the mechanism of energy dissipation discussed in the Tomlinson model is applied only to atomic-scale friction.

There are several versions of the Tomlinson model, presented in slightly different ways $[10,11]$. These versions have been employed to explain the atomic stick-slip behavior between the tip and sample, and the velocity dependence of friction observed in AFM experiments $[12,13]$. The Tomlinson model extended to a finite temperature shows that if there is an energy barrier $\Delta E$ between two neighboring minima in potential energy, the tip may still be able to jump from one energy valley to the next due to thermal activation. The probability for the tip to jump (or not to jump) depends on the sliding velocity and the exponential of $\Delta E / k_{\mathrm{B}} T$, which leads to a logarithmic dependence of friction on velocity. This confirms Tabor's proposal in his lecture at the NATO conference that the behavior of speed-dependent friction may be understood in terms of the stress-aided rate theories developed by Eyring.

A similar logarithmic dependence between the force needed to break a molecular bond and the velocity to pull the bond is known to scientists studying adhesion. Suda revealed the connection between the two processes, where velocity dependence in friction can be derived independently from the rupture dynamics [14]. This means that the Tomlinson model is essentially consistent with rupture dynamics when modeling atomic-scale friction. More importantly, Suda's analysis offered an insight into the relation between friction and adhesion hysteresis, which will be further discussed in Section 4 .

Another extension to the Tomlinson model was proposed recently by Huang and coworkers [15]. In this so-called composite oscillator model, there are two rows of oscillators connected with each other in both vertical and lateral directions, and the lower harmonic potential is replaced by oscillators arranged similarly to those in the top system.

Researchers are still able to learn important lessons from the Tomlinson model today. For example, it is natural to infer from the model that the extent of energy dissipation and the magnitude of average friction force depend on two factors, the corrugation of interfacial potential and the stiffness of the surface bond. It also sheds light on the study of superlubricity where a frictionless state can be achieved because of lack of energy dissipation if the spring stiffness is strong enough in comparison to the interfacial interaction, as will be discussed further in later sections. In addition to the Tomlinson model, there are two independent developments that contribute greatly to the study of atomic-scale friction, as summarized in the following sections.

\section{Role of commensurability-the Frenkel-Kontorova model}

\subsection{Frenkel-Kontorova model}

The FK model involves a system shown in Fig. 2 where a one-dimensional chain of atoms connected by springs of average length $a$ interacts with a harmonic potential in period $b$ [16]. The model was employed at first to study dislocation in crystals, but it soon proved useful in studying the mechanism of friction such as the origin of static friction and effect of structural commensurability.

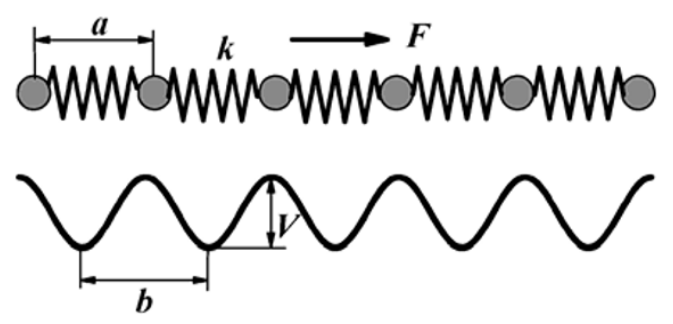

Fig. 2 Schematic of the Frenkel-Kontorova model. 
The dimensionless equation of motion for the atomic chain can be written as

$$
\begin{aligned}
& \ddot{x}_{j}+\gamma \dot{x}_{j}=\left(x_{j+1}-2 x_{j}+x_{j-1}\right)-\lambda \sin 2 \pi x_{j}+F \\
& j=1,2, \ldots, N
\end{aligned}
$$

where $x_{j}$ is the position of the $j$ th atom, $\gamma$ denotes the internal damping coefficient in the atomic chain, $\lambda=V / k$ represents a normalized potential strength equal to the magnitude of the sinusoidal potential divided by spring stiffness $k$, and $F$ is an external driving force.

The chain is at the ground state when all the atoms are resting in the energy valleys, i.e., their equilibrium positions, which deviate from their initial position by a small shift $\xi_{j}$,

$$
x_{j}=x_{B}+a_{0} j+\xi_{j},
$$

where $a_{0}$ is the initial length of the spring and $x_{\mathrm{B}}$ is the mass center position of the chain. Aubry [17] defined a hull function $g(x)$ for describing the shift,

$$
\xi_{j}=g\left(x_{B}+a_{0} j\right)
$$

If the normalized strength $\lambda$ is small, the hull function is analytic where an infinitesimal force would be able to make the chain slide adiabatically. However, when the strength exceeds a critical value $\lambda_{c}^{S}, g(x)$ is no longer analytic and becomes a discontinuous function. This is called the breaking of analytics or Aubry transition. The transition leads to a pinning state where the atomic chain is locked by the harmonic potential so that a finite force must be applied in order to initiate a slide of the chain. This is the first time researchers were able to predict static friction mathematically in terms of the breaking of analytics of a well-defined function.

Great efforts have been devoted to explore the dynamic response of the system by solving the equation of motion at a finite sliding velocity. In the over-damping condition when the damping coefficient $\gamma$ is large, Eq. (2) gives rise to a periodic solution [18], and the system exhibits rich dynamics as damping decreases. In addition to the periodic solutions, there are quasi-periodic or even chaotic solutions, depending on the parameters $\gamma, \lambda$, and the sliding velocity $v$ $[19,20]$. It is important to note from the solutions that the vibrations of the atomic chain and particularly resonance at certain velocities may introduce a possible mode of energy dissipation for sliding at a finite velocity.

Compared to a real system, both the FK model and the Tomlinson model seem to miss something important. In the former, the atoms are not connected to a support representing the bulk of a sliding body whereas in the latter, the lateral interactions between the oscillators have been ignored. In consideration of the atomic interactions in a real interfacial system, it would make more sense to couple the two models together, leading to the Frenkel-Kontorova-Tomlinson (FKT) model proposed by Weiss and Elmer [21, 22].

Because the FK and FKT models involve two periodic structures interacting with each other, they represent an effective approach for analyzing the role of structural commensurability in energy dissipation.

\subsection{Role of commensurability}

Commensurability describes a length-scale correlation between two interacting objects composed of periodic structures with periods $a$ and $b$ (see Fig. 2, for example). The structure is called commensurate when the period $a$ is a simple rational fraction of $b$, or the ratio $c=a / b$ is a rational number; otherwise, the structure is incommensurate.

The effects of commensurability on static friction have been studied using the FK or FKT model [17, 21]. Numerical solutions of the static friction force $F_{\mathrm{S}}$ under different values of $a / b$, for example, were obtained by Weiss [21], as plotted in Fig. 3 against the strength $\lambda$. It can be seen that in the incommensurate case where the ratio $a / b$ equals the irrational number $(\sqrt{5}-1) / 2$ (the golden mean), $F_{\mathrm{S}}$ remains zero until the strength exceeds a critical value, $\lambda>\lambda_{c}^{S}$, as discussed in Section 3.1. The critical strength decreases, accompanied by a monotonous rise in friction as the ratio takes the values of $3 / 5,2 / 3,1 / 2$ and so forth until $\lambda_{c}^{S}=0$ for $a / b=1$ when the system becomes perfectly commensurate.

Similar dependence on commensurability was observed for kinetic friction in the quasi-static limit of $\mathrm{v} \rightarrow 0$. In the framework of the FK or FKT model, kinetic friction can be determined by solving the equation of motion. As long as $\lambda$ is small, there is a unique solution for the equation. In this case, the 


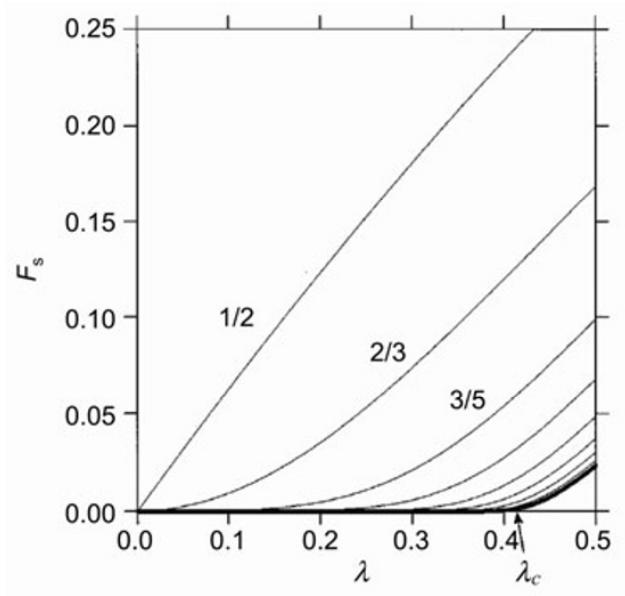

Fig. 3 Static friction as a function of and for a sequence of rational values of $c$ (thin lines), which converges to the golden mean (thick line) [21].

friction is a conservative force fluctuating around zero. Its average is zero (i.e., $F_{K}=0$ ). There is a second critical value for the potential strength, $\lambda_{c}^{K}$, above which the solution is no longer uniquely defined and metastable states appear. As the system approaches a point of bifurcation, the atoms have to jump from one metastable state to a more stable position so that the system is rearranged into a new configuration, accompanied by energy dissipation. The average friction and the value of $\lambda_{c}^{K}$ were numerically calculated by Weiss and Elmer [21], which revealed the dependence of kinetic friction on the commensurability.

The diverse frictional behavior in a commensurate or incommensurate system results from the difference in energy dissipation, which may be understood in terms of the Tomlinson model discussed in Section 2. For two lattices in contact and in relative motion, each atom on one lattice surface is subjected to interaction from the opposite body, described by a harmonic potential, and the total potential energy applied to the entire surface is obtained by superposing the interaction on a single atom. In the incommensurate case, the superposed potentials cancel each other, leading to a total energy with little corrugation. It is the disappearance of energy corrugation that prevents energy dissipation and gives rise to very low friction.

As an example, Fig. 4 gives the plot of shear stress vs. sliding distance obtained from MD simulations for two sets of self-assembled monolayers (SAMs) in relative sliding [23]. Fig. 4(a) shows the results from a
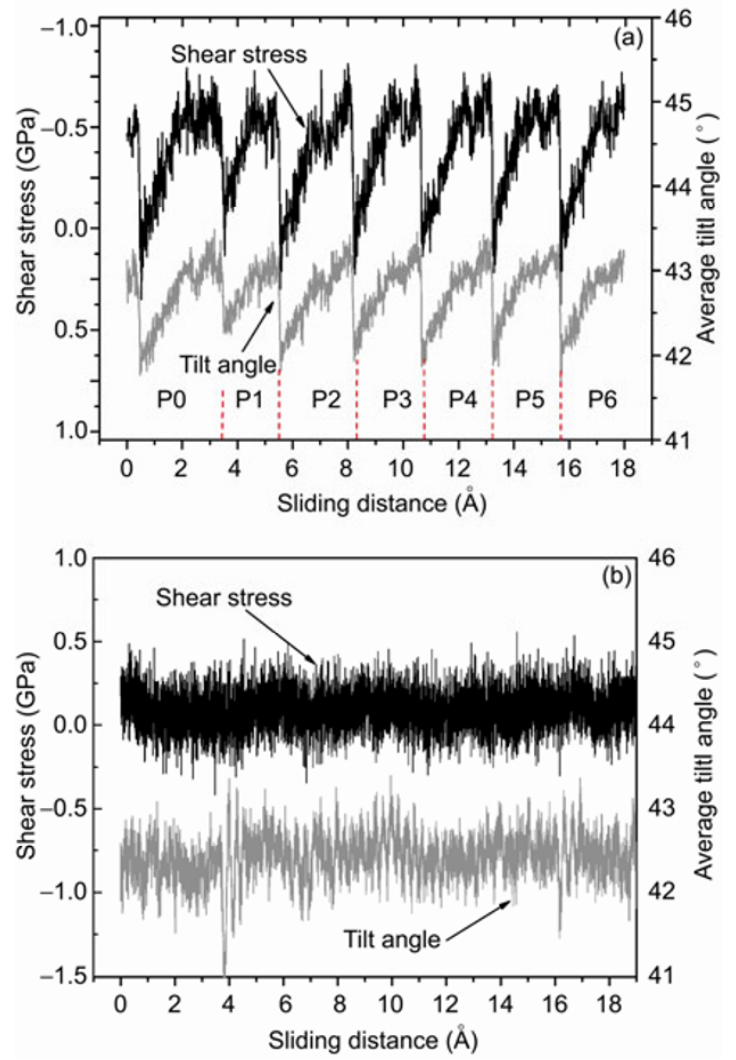

Fig. 4 Shear stress (dark line) and molecule tilt angle (gray line) from MD simulations for commensurate (a) and incommensurate SAMs (b) [23].

commensurate system where the upper and lower monolayers are perfectly aligned, and Fig. 4(b) shows results from the case where one of the monolayers has been turned by $90^{\circ}$ so that the system becomes incommensurate. The shear stress in the commensurate system presents a distinct stick-slip pattern, while in the incommensurate case, the stick-slip disappears with only thermal fluctuations left in the curves, and the average shear stress is five times smaller than that in the commensurate case.

The first experimental evidence for the effect of commensurability was found in a study using a QCM in 1991 when Krim reported that solid monolayers of krypton sliding on gold exhibited five times less friction than liquid monolayers of krypton sliding on gold did [24]. The observed behavior indicating solids are more slippery than liquids was difficult to explain at first. It was discovered a few years later that the higher friction of liquid layers can be attributed to the fact that the liquid layers are more 
flexible and therefore slightly more commensurate with the underlying surface than the solid layers are [25]. More direct and convincing evidence came from an experiment measuring the friction between an AFM tip and a highly oriented pyrolytic graphite (HOPG) sample. The experiment revealed a dependence of friction on sliding direction, as shown in Fig. 5, where the measured friction is plotted as a function of rotation angle of the sample [26]. During the sliding, a small piece of graphite was transferred to the tip so that friction is in fact occurring between the graphite sheet adhering to the tip and the HOPG substrate. A rotation of the sample would cause a change in commensurability between the sheet and substrate. As a result, the friction remains very low when they are incommensurate unless the rotation causes the substrate to be commensurate with the sheet, resulting in high friction.

There is an unsolved mystery regarding the effect of commensurability. The predicted frictionless state in incommensurate systems does not exist in macroscopic reality although almost all surfaces in macroscopic contacts will always be incommensurate [25]. Even two surfaces of the same crystal are incommensurate unless they are aligned perfectly. Several hypotheses have been proposed to explain the mystery. For example, the strong interfacial interaction may cause local transitions from incommensurate to commensurate state, which is energetically more favorable, and the presence of lubricant molecules confined between two surfaces may modulate the system into a commensurate condition. The role of lubricant molecules will be discussed in the next section.

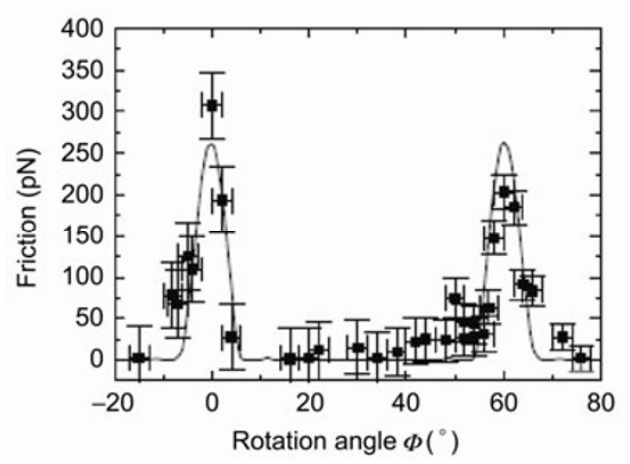

Fig. 5 Friction force between an AFM tip and a HOPG sample plotted as a function of rotation angle of the sample [26].

\section{Evaluating shear strength at the interface - the cobblestone model}

A cobblestone model was proposed first by Tabor [27] and developed further by others [28] to explain the friction of two solid surfaces sliding past each other in the absence of wear. The model suggests that the process of friction is similar to pushing a cart over a road of cobblestones, as shown in Fig. 6, where the cartwheels represent the molecules of lubricant or of the upper solid body, and the cobblestones represent atomic roughness of the lower surface [29]. A certain lateral force $F$ is required to raise the cartwheel against the attractive surface interactions in order to initiate motion. Assume that the normal distance between the two surfaces increases by a small amount $\Delta D$, meanwhile the upper surface moves forward a distance $\Delta d$ along the lateral direction. The work done by the lateral force $F$ to initiate sliding has to be balanced with the work required to overcome the force of adhesion $F_{\text {ad, }}$ which can be estimated in terms of the surface energy change when the normal distance increases from $D=D_{0}$ to $D=\left(D_{0}+\Delta D\right)$.

$$
\Delta d \times F=\Delta D \times F_{\text {ad }}=2 \gamma A\left(1-\frac{D_{0}^{2}}{\left(D_{0}+\Delta D\right)^{2}}\right) \approx 4 \gamma A \frac{\Delta D}{D_{0}}
$$

where $\gamma$ denotes the surface energy and $A$ is the area of contact.

From the point of view of energy dissipation, however, it is inappropriate to balance the frictional work only with the energy required for surface separation because the process of sliding consists of a series of events of surface separation and approach. A

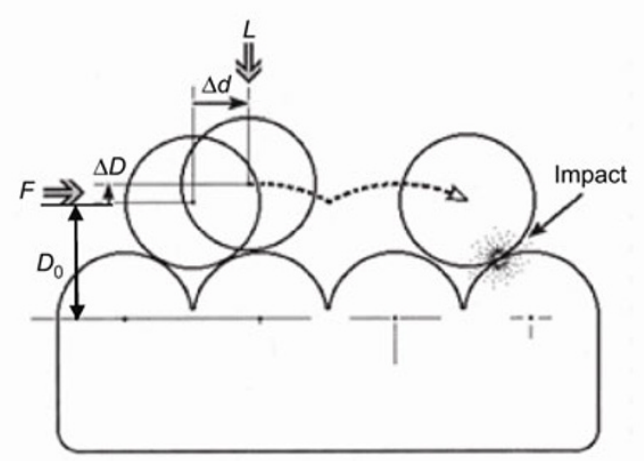

Fig. 6 Schematics for the cobblestone model. 
part of the work expended on separating the surfaces will be regained during the process of approach. If the energy to separate surfaces is fully recovered when they approach, there will be no energy dissipated and the average friction force will be zero. With this consideration, the work done by the friction force should equal to the energy loss in a cycle of surface separation/approach, and Eq. (4) is be rewritten as

$$
\Delta d \times F=4 \Delta \gamma \cdot A \frac{\Delta D}{D_{0}}
$$

where $\Delta \gamma=\left(\gamma_{\mathrm{s}}-\gamma_{\mathrm{r}}\right)$ is the difference in energies for surface separation and approach. As a result, the shear strength at the interface can be evaluated by the following equation.

$$
S_{c}=\frac{F}{A}=\frac{4 \Delta \gamma \cdot \Delta D}{D_{0} \cdot \Delta d}=\frac{4 \gamma \varepsilon \cdot \Delta D}{D_{0} \cdot \Delta d}
$$

In the above equation, a coefficient of dissipation defined as $\varepsilon=\Delta \gamma / \gamma$ is introduced to specify that a fraction of the energy is lost every time the surfaces experience a cycle of separation/approach.

Some important outcomes of the cobblestone model are discussed below.

(1) The model gives an estimation of the shear strength for the monolayers at the interface or for thin liquid films confined between two surfaces. For a typical hydrocarbon surface, $\gamma \approx 25 \times 10^{-3} \mathrm{~J} / \mathrm{m}^{2}$, and other typical values are set as $\Delta D=0.5 \AA, D_{0}=2 \AA$, $\Delta d=1 \AA$, and $\varepsilon=0.1$. Eq. (6) predicts

$$
S_{c} \approx 2.5 \times 10^{7} \mathrm{~N} / \mathrm{m}^{2} .
$$

This compares very well with the typical experimental value of $2 \times 10^{7} \mathrm{~N} / \mathrm{m}^{2}$ calculated for a hydrocarbon surface sliding in air or separated by a one-molecule layer of cyclohexane [28].

(2) The model provides a theoretical frame for understanding the relation between friction and adhesion hysteresis. In contrast to common belief, the experiments in SFA conducted by Israelachivil and coworkers [30] reveal that friction is correlated with the adhesion energy hysteresis rather than the adhesion energy itself. The cobblestone model explains that due to the presence of atomic-scale roughness, a sliding motion is always accompanied by surface separation and approach, and it is the energy difference between the two motions (or the energy hysteresis) that determines the magnitude of friction. Consequently, the shear strength predicted by Eq. (6) is in direct proportion to $\Delta \gamma$. As revealed by Suda [14], the relationship between friction and adhesion hysteresis can be derived from rupture dynamics. If friction is considered as the force needed to break interfacial bonds, it can be expressed as $F_{\mathrm{f}}=(1 / d)\left(N k_{\mathrm{B}} T \ln \tau_{1}-\right.$ $\left.N k_{\mathrm{B}} T \ln \tau\right)$, where $\tau_{1}$ and $\tau$ are the bond lifetime at equilibrium and at the transition state, respectively. Assuming that $\gamma_{\mathrm{A}}$ and $\gamma_{\mathrm{R}}$ are equivalent to $\left.-\left(N / \pi r^{2}\right)\right) k_{\mathrm{B}} \ln \tau_{1}$ and $\left.-\left(N / \pi r^{2}\right)\right) k_{\mathrm{B}} \ln \tau$, respectively, one finally obtains $F_{\mathrm{f}}=\left(\pi r^{2} / d\right)\left(\gamma_{\mathrm{R}}-\gamma_{\mathrm{A}}\right)$.

(3) Introduction of the factor $\varepsilon$ represents progress toward clarifying the amount and mode of frictional energy dissipation. It has been long recognized that friction is accompanied by accumulation and release of mechanical energy but there are few clues as to what percentage of the energy is converted into heat. The factor $\varepsilon$ gives an estimation for the fraction of the energy to be lost irreversibly. Moreover, the cobblestone model seems to suggest that the energy fraction is lost through the impact between the cartwheel and cobblestone (see Fig. 6). Tabor himself mentioned once that he was not totally pleased with this model because it gave no indication of the mode of energy dissipation. In reviewing this model, however, we realized that the model has in fact related the energy dissipation to a specific mechanism-adhesion hysteresis, which has not been expressed explicitly in other models. The adhesion hysteresis as a mechanism of frictional dissipation may be useful in describing applications such as boundary lubrication and friction of viscoelastic materials. This explains why the cobblestone model is widely accepted in the community of polymer tribology.

\section{Progress of energy dissipation - phononic and electronic damping}

With the revelation that energy dissipation initiates from mechanical instability, the next questions involve the dissipation progress after interfacial atoms start to vibrate. Is the energy dissipated permanently into heat or is it transformed into different forms of energy? These questions are discussed in the following sections. 
From the phononic model system shown in Fig. 7(a), a general description of the process of energy dissipation can be summarized as follows [31]: A nanoparticle consisting of $N$ atoms (representing, for example, an AFM tip or a lubricant molecule) is coupled to the surface of object 1 . The nanoparticle and the object 1 surface slide together over the surface of a lower sample (object 2) under a constant loading force $F_{N}$. In the initial stick stage, the nanoparticle is elastically deformed, namely, its atoms are displaced whereby the potential energy of the particle is increased by $\delta V_{T}$. Once the nanoparticle arrives at a critical point $M$, slip occurs when the nanoparticle jumps to an adjacent energy valley. In the course of slip, the displaced atoms of the nanoparticle are suddenly released and they start to vibrate around their equilibrium positions.

\subsection{Generation of excess phonons}

In the framework of phonon theory, the release of accumulated energy $\delta V_{T}$ at the time of slip means that the energy is expended irreversibly to excite phonons above the occupation number present at ambient temperature $T_{0}$. The excess phonons will be damped or will decay into a new state of equilibrium at a higher temperature $T$. Denoting the occupation numbers at $T$ and $T_{0}$ as $n_{q}$ and $n_{q}^{o}$, respectively, the excess phonon distribution is expressed as,

$$
\Delta n_{q}=n\left(\Omega_{q}, T\right)-n^{o}\left(\Omega_{q}, T_{0}\right)
$$

Here, $q$ denotes the mode of the phonon, $\Omega_{q}$ is the phonon frequency in mode $q$, and $T>T_{0}$. The total number of excess phonons covering all possible modes can be written as $\Sigma_{q} \Delta n_{q}$. Since the nanoparticle

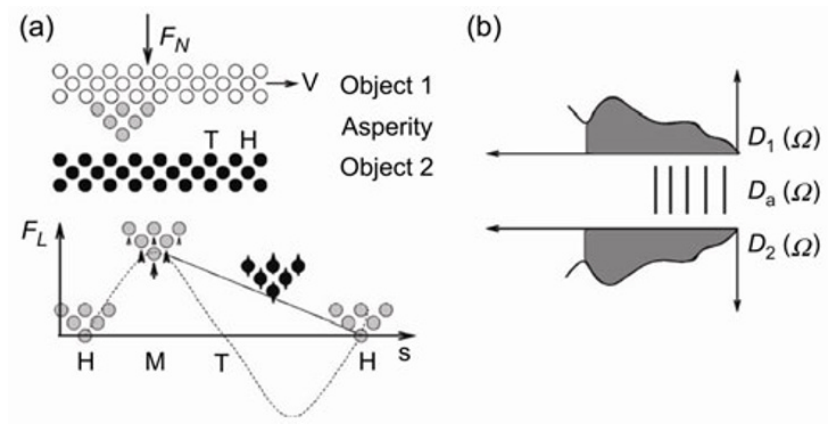

Fig. 7 System used for modeling phononic damping [31]. contains only a small number of atoms, it has a discrete frequency distribution with relatively large spaces between the frequencies, $D_{a}(\Omega)=\Sigma_{q} \delta\left(\Omega-\Omega_{q}\right)$; meanwhile, the frequency distributions (or the densities of state) of the objects, $D_{1}(\Omega)$ and $D_{2}(\Omega)$, are quasicontinuous as shown in Fig. 7(b).

The decay or damping of the excess phonons, $\Sigma_{q} \Delta n_{q}$ may occur in various ways. For example, the phonons may be damped by (1) phonon-phonon interactions in the nanoparticle, (2) interactions between the nanoparticle and adjacent objects, or (3) phononelectron interactions. In the following, we start with the first and second mechanisms, i.e., phononic damping, and electronic damping will be discussed in Section 5.4.

\subsection{Internal damping}

If the nanoparticle is isolated from all adjacent objects, the phonons are damped solely by the phononphonon interaction occurring inside the particle, i.e., by the internal damping, in which the anharmonic coupling between phonons causes the vibration energy of a phonon to be translated to other phonon modes. If the nanoparticle is small enough or the anharmonic terms are weak, there is a possibility that the phonons will not be damped by internal anharmonic interactions so they remain in discrete modes with no energy dissipation.

Sokoloff examined energy dissipation in a mesoscopic solid represented by a linear atomic chain with its atoms interacting through harmonic and anharmonic forces with one end of the chain forced to vibrate harmonically. This model represents the interactions when the solid is sliding over another substrate [32]. The results show a possible frictionless sliding for the atomic chain in the sense that the internal energy of the system does not increase with time. The study also reveals a critical size of the system beyond which there is a transition from nondissipative to dissipative behavior. A similar size dependence of friction was reported by Kajita and coworkers [33] in analyzing a two dimensional crystal where friction was found to increase with the number of atomic layers, which was explained in terms of the contributions from bulk atoms to internal damping activity. 


\subsection{Interfacial damping}

If the nanoparticle is coupled weakly with an adjacent object, the coupling will also cause phonon decay, known as interfacial damping. The number of phonons in mode $q$ will vary with time, following the equation below.

$$
\frac{\mathrm{d} \Delta n_{q}}{\mathrm{~d} t}=-\Gamma_{q}
$$

$\Gamma_{\mathrm{q}}$ is the decay rate of phonons from mode $q$ to the object, also known as the coupling constant.

The deformation energy of the nanoparticle accumulated during stick, $\delta V_{T}$, is converted into excitation energy of the phonons during slip, so the energy can be written in phononic form as

$$
\delta V_{T}=\sum_{q} \delta V_{q}=\sum_{q} \hbar \Omega_{q}\left(\Delta n_{q}+\frac{1}{2}\right)
$$

where $\delta V_{q}$ denotes the phononic energy in mode $q$. Combining Eqs. (8) and (9) leads to an estimation that the energy $\delta V_{T}$ decays in exponential manner with a dissipation rate depending on the factors related to the number of phonons, the strength of coupling, and the density of state of the coupled objects. For the system shown in Fig. 7 and a given damping coefficient, the energy $\delta V_{T}$ almost vanishes within 100 ps.

Phononic description theory has not advanced sufficiently to provide an accurate prediction of energy dissipation. However, an important lesson we have learned from the analysis is that dissipation is a process in which the state density of phonons transits from a few discrete modes into a widespread and continuous distribution. Both internal and interfacial damping contribute greatly to the energy dissipation in the sense that they help phonons to transit from one particular mode to other modes. The question as to whether phononic friction is dominated by internal or interfacial damping remains unclear, but is expected to depend on the specific frictional system.

\subsection{Electronic damping}

Electronic damping may contribute significantly to frictional dissipation for sliding on conductive or metallic surfaces. The mechanism involves excitation of electron-hole pairs as a result of electron-phonon coupling, and progress has been made in evaluating the electronic damping by measuring the "slip time" or the increase in electrical resistivity.

When a microscopic particle or a molecule slides on a substrate, the friction force acting on the particle can be characterized by a "slip time" $\tau$, corresponding to the time elapsed before the speed of the particle falls to 1/e of its original value. Slip time is inversely proportional to the amount of frictional damping. If both phononic and electronic damping are considered, the slip time can be written in an additive form, including the separate contributions from phonon $\tau_{\mathrm{ph}}$ and electron $\tau_{\text {el }}$ slip times.

$$
\frac{1}{\tau}=\frac{1}{\tau_{\mathrm{ph}}}+\frac{1}{\tau_{\mathrm{el}}}
$$

Slip times for various monolayers and bilayers adsorbed on metal substrates have been measured using a QCM by Krim and coworkers [24]. It is reported that the measured slip times of Xe on $\mathrm{Ag}$ are in perfect agreement with the results of $\mathrm{MD}$ simulations with no electronic damping [25]. This seems to suggest that the phononic damping is dominant, but simulations conducted by other investigators have lead to a different conclusion [34]. This has caused a debate regarding the importance of the electronic contribution to friction, as will be discussed later in this section.

The effect of electronic damping has been observed in an experiment involving $\mathrm{N}_{2}$ adsorbed on lead [35], which reveals a temperature dependence of the slip times as shown in Fig. 8. As the temperature decreases below the superconducting transition temperature $T_{\mathrm{c}}$ the shear stress drops rapidly and the slip time shows a sharp increase. The sharp decrease of friction was considered a proof of the fact that electronic friction exists in normal conditions but disappears at temperatures below $T_{\mathrm{c}}$ due to superconductivity.

It has been shown by Persson [36] that the damping of molecules moving on a metal substrate is equivalent to an increase in the electrical resistivity of the substrate that occurs when molecules are adsorbed on the surface. As a result, the electronic slip time of a sliding particle can be calculated from the increase in the resistivity caused by adsorption of the particle. This enables one to evaluate the coefficient of electronic 


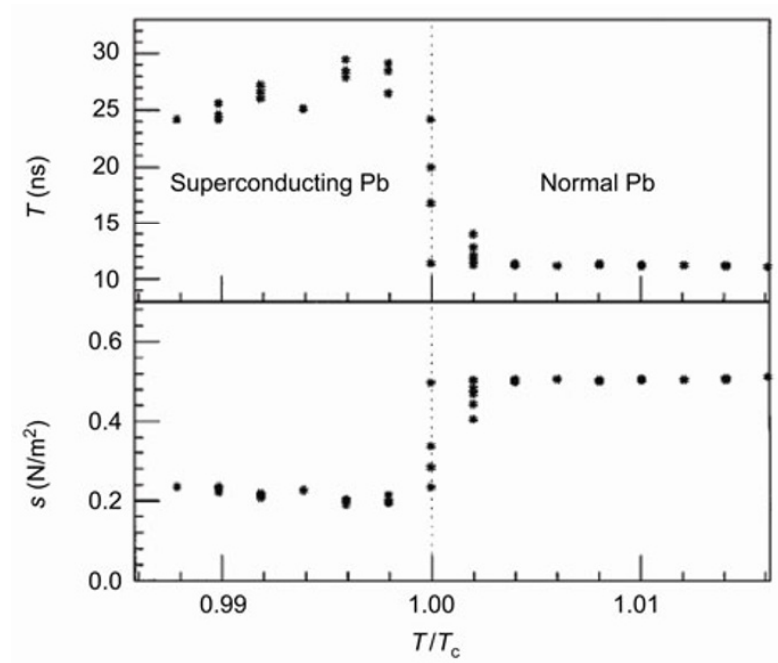

Fig. 8 Temperature dependence of slip time and shear stress for $\mathrm{N}_{2}$ adsorbed on lead [35].

damping $\eta_{\mathrm{el}}$ in terms of the changes to resistivity as a function of adsorption coverage. The values of $\eta_{\mathrm{el}}$ for several different molecules adsorbed on Ag range from $3 \times 10^{8}$ to $3 \times 10^{9} \mathrm{~s}^{-1}$, which are much smaller in comparison to the coefficient of phononic damping $\eta_{\mathrm{ph}}$ deduced from the He-atom scattering measurement for hydrocarbons adsorbed on $\mathrm{Cu}(100)$.

However, the role of electronic friction remains an open question. Different investigators have carried out several studies but results have been inconsistent. As mentioned above, MD simulations without considering any electronic effect produce friction curves that agree well with those from the QCM experiments, suggesting that the contribution from electronic damping is insignificant [25]. Experiments by Fois and coworkers [37] for a system similar to that in Ref. [35] found that the $\mathrm{N}_{2}$ adsorbate sticks to the $\mathrm{Pb}$ substrate at the superconductivity temperature and does not show any slip, which is not consistent with Krim's observations. Besides, the abrupt transition in friction coefficient observed in Ref. [35] is in conflict with the prediction of the Bardeen-Cooper-Schrieffer (BCS) theory commonly accepted to describe superconductivity at low temperatures. This has caused a debate regarding the reproducibility of the results reported in Ref. [35]. Recently, a measurement of friction between a pendulum-type AFM probe and $\mathrm{Nb}$ films was carried out [38]. The results showed a smooth transition of friction across the critical temperature of superconductivity, which is consistent with the BCS theory. A close examination confirms that the dependence of friction on tip-film distance at the temperature above the critical point $T_{\mathrm{c}}$ is consistent with the prediction for a two-dimensional electron system while dependence below $T_{\mathrm{c}}$ follows the law of phononic friction. This clearly shows the existence of electronic friction but further studies are certainly needed to clarify the contribution of electronic damping to friction.

Below we discuss two types of energy dissipation that appear to be different from those discussed above, but at a fundamental level they belong to the same category as phononic dissipation.

(1) Friction-induced sound

The energy of elastic deformation accumulated in the stick phase may be released by emitting elastic or acoustic waves directly into the air instead of being converted into heat. As an example, while playing a violin, the stick-slip friction between the bow and the string is transformed to sound without significant heat generation. In contrast, brake noise in automobiles is an extreme example where sound is accompanied by a large amount of heat generated at the contact surfaces. Friction-induced sound and noise has become a major research subject due to its importance in engineering applications [39]. Generally, the energy release through sound wave emissions should be cataloged as a special form of phononic dissipation [40], but the acoustic radiation in dynamic friction can be analyzed more specifically by an approach similar to that in electromagnetism [41]. Radiative damping will produce a force to resist motion where amplitude increases with frequency according to a cubic law, much faster than that of viscous damping, and the power spectrum of surface displacements will decay at a rate proportional to $\omega^{6}$ as confirmed in a recent experiment, where $\omega$ is the frequency of dynamic friction [41].

(2) Thermoelastic damping

The vibrations of an elastic beam or a microscopic resonator, no matter how carefully designed, will decay to a complete stop as a result of energy dissipation. The mechanism of energy dissipation involved here is called thermoelastic damping, which couples the elastic deformation directly to a local temperature rise. The strength of the coupling can be described by 
a single microscopic parameter, namely, the material's thermal expansion coefficient [42]. In atomic-scale friction accompanied by stick-slip motion, there is considerable elastic strain accumulated in the stage of stick so that thermoelastic damping has to be a possible mechanism of energy dissipation. In the phononic description, thermoelastic damping is caused by interactions between the acoustic mode (vibrations) and a surrounding bath of thermal phonons, so it is a special form of the internal damping discussed in Section 5.2.

\section{Contributions from other mechanisms of energy dissipation}

The subjects we have discussed so far concern a specific mechanism of energy dissipation that transforms mechanical energy into heat when two solid surfaces slide past each other. The mechanism involves the accumulation and release of deformation energy, atomic vibration at the interface, and decay of the vibration energy into heat. However, other mechanisms, such as the viscoelastic relaxation and material wear, may also contribute to the energy transformation in the course of sliding, as discussed in this section.

\subsection{Viscoelastic relaxation}

For a solid obeying linear elasticity subjected to a constant elastic strain, the strain will be constant with time, but in the case of a liquid obeying linear viscosity, the strain will be released immediately due to its incapability to store energy. The situation becomes more complicated for a viscoelastic material in which the elastic strain will be gradually released within a relaxation time that depends on material properties. The reason for raising this question is a concern regarding the possibility that viscoelastic relaxation may serve as a mechanism of frictional dissipation, especially for viscoelastic materials such as rubbers, polymers, and organic lubricant films. Evidence for such a mechanism of dissipation was found in a fretting friction test involving polymers [43], where the origin of the dissipation was attributed to viscoelastic loss within the contacting asperities. This was based on observations that the ratio between the out-of-phase and in-phase displacements was of the same order as the loss angle tan $\delta$ for the bulk polymer under test. Lei and Leng [44] also found dissipation associated with viscoelastic relaxation in MD simulations involving liquid argon confined between solid walls. They reported that about $60 \%$ of total frictional work was converted into heat at the time of slip and the remaining $40 \%$ was released more slowly into the solidified films through various channels, including relaxation.

While frictional dissipation for incompliant materials has been described by the Tomlinson model in terms of excitation of atomic vibrations, the sliding of compliant and viscoelastic materials can be also viewed as an activated process, in which molecules experience shear-induced de-bonding and relaxation that result in energy dissipation. The frictional processes involving molecule activation and relaxation can be described by a simple model based on Eyring's theory of reaction rates.

By plotting the friction force measured between an AFM tip and a polymer substrate as a function of logarithmic sliding velocity, one obtains a bell-shaped curve as shown in Fig. 9 if the shear rate matches the material intrinsic relaxation time [45].

Note that for curves measured at different temperatures, there are horizontal shifts $a_{T}$ from the curve obtained at the reference temperature as shown in Fig. 9. If the data are treated based on the theory of time-temperature equivalence, one will get a master curve independent of temperature. The velocity and temperature dependence of friction and associated energy dissipation are a central theme in the study of friction for viscoelastic materials.

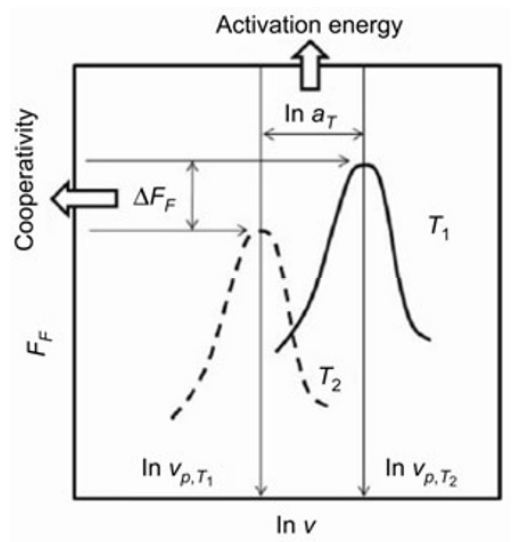

Fig. 9 Friction-velocity curves showing a bell-shaped dependence with horizontal and vertical shifts [45]. 
Due to the time-temperature equivalence, the horizontal shifts provide the means to determine the apparent activation energy $E_{a}$

$$
E_{a}=-R\left[\frac{\partial \ln \left(a_{T}\right)}{\partial(1 / T)}\right]_{P}
$$

The thermal shift and the shape of the frictionvelocity curve provide an energetic signature for the dissipation mechanism. The bell-shaped dependence originates from the competition between two time scales, namely, (i) the extrinsic drive time, and (ii) the intrinsic material response time. The friction force increases or decreases with increasing sliding velocity depending on whether the extrinsic time leads or trails the material response time. Friction force $F_{F}(v)$ peaks at a critical velocity $v_{\mathrm{p}}$ when the two processes occur on comparable time scales. The product between $v_{\mathrm{p}}$ and the relaxation time $\tau_{\alpha}$ leads to a "dissipation length" that provides an estimation for the size of the region in which energy dissipation occurs [46].

\subsection{Contributions from wear}

The concept of friction without wear confuses engineers somewhat, wondering how it could happen in reality. On the contrary, the relation between friction and wear is easy to understand. There is a common belief in the tribology community that plastic deformation and wear consume energy so they are responsible for the generation of friction. This is true in some cases. The ploughing of a hard indenter in a soft material is a simple example that relates friction force directly to the plastic deformation of the ploughed material [2].

In general cases of sliding, friction is accompanied by material damage so that in addition to the energy dissipation via atomic vibrations, a portion of frictional work is expended to cause plastic deformation, microcracks, and wear [47]. The evidence for a linear relationship between measured wear volume and energy dissipation has been found in experiments [48], as illustrated in Fig. 10. The wear volume measured in a test for a TiN monolayer sliding against alumina balls is plotted as a function of dissipated energy. A number of other models have been proposed to relate wear rate with energy dissipation [49].

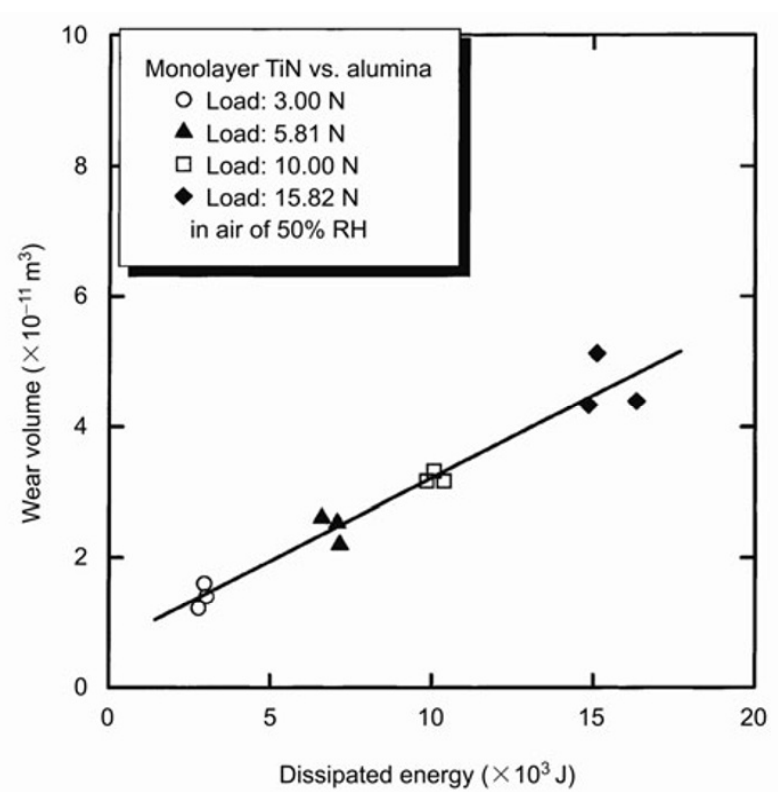

Fig. 10 Linear relation between wear volume and dissipated energy from sliding wear tests of a TiN monolayer against alumina balls [48].

However, the energy-based wear models seem to suggest that all or most of frictional work is dissipated in the form of wear. This implicit assumption may be acceptable for fretting wear, but in normal sliding it is estimated that only a small portion of frictional work (less than $10 \%$ ) will be stored in material in the form of structural distortion, and then be converted into wear $[50,51]$. The estimate is supported by the fact that the wear rate in normal sliding is about 10 times lower than that observed in fretting tests for the same material combinations [48]. Nevertheless, the big question regarding the division between the two dissipation mechanisms, namely wearless heating and wear-related energy loss, remains unanswered.

The above discussions also explain why we have to focus on wearless friction. There are two reasons: (i) Wearless friction is a major part of the energy dissipation in normal sliding, and (ii) wearless dissipation is more difficult to understand and resolving the mystery is a challenging mission for us in tribology to accomplish.

While focusing on energy dissipation in wearless friction, we noted that research on atomistic wear has increased recently because of its connection to nanofabrication. It is reported that in processes where material is removed atom by atom, wear does not 
obey the Archard equation but can be described by a model based on the breaking of individual bonds [52].

\section{Energy dissipation in multilayer graphene-An example}

As an example, we will examine a specific process of energy dissipation on the basis of the results of MD simulations performed recently by $\mathrm{Xu}$ [53] for a system consisting of multilayer graphene, with the top graphene layer being forced to move at constant velocity while the bottom layer remains fixed. The energy components and their time evolutions are displayed in Fig. 11. $D_{\mathrm{e}}$ denotes the work done by the external lateral force that drives the system, $P_{\text {ae }}$ is the potential energy of the system, $K_{\mathrm{ae}}$ defines the vibrational energy of all atoms, and $K_{\mathrm{e}}$ is the kinetic energy caused by the center-of-mass motion of graphene layers. The left and right parts of the figure correspond to the results from a three- and a five-layer system, respectively.

The results shown in Fig. 11 allow us to analyze the details of energy dissipation occurring in the multilayer graphene system, as summarized below.

(1) Energy input and accumulation

In the stick stage, the top graphene layer is pulled by an external lateral force to move it forward, and the work done by the external force is input to the system and stored in the form of potential energy $P_{\text {ae }}$. As a result, both $D_{\mathrm{e}}$ and $P_{\mathrm{ae}}$ increase in a synchronous manner, as shown in Fig. 11.

(2) Transformation of energy to atomic vibration and layer translation

In the slip stage, the potential energy drops rapidly while $K_{\mathrm{ae}}$ and $K_{\mathrm{e}}$ increase, indicating that a part of the stored potential energy is converted to atomic vibrations and the mass-center motion of the graphene layers. The increase in $K_{\mathrm{ae}}$ and $K_{\mathrm{e}}$ occurs in very short time, a few picoseconds according to the curves in Fig. 11, and is accompanied by a sharp decrease of $P_{\mathrm{ae}}$ in the same time. The mechanism for the transformation of energy from $P_{\mathrm{ae}}$ to $K_{\mathrm{ae}}$ and $K_{\mathrm{e}}$ can be well understood in terms of the Tomlinson model and phononic damping discussed in previous sections.

(3) Further transformation of the translational energy $K_{\mathrm{e}}$

It can be seen from Fig. 11 that during the time interval from the instant of slip to the full development of the next stick, there is a visible decrease in the translational energy $K_{\mathrm{e}}$, especially for the five-layer system, but no detectable increase in vibration energy $K_{\mathrm{ae}}$. This indicates that the kinetic energy for the mass-center motion of graphene layers has been further transformed into other forms of energy with at least two possibilities: either $K_{\mathrm{e}}$ is further converted to atomic vibrations and heat, or a part of it is returned
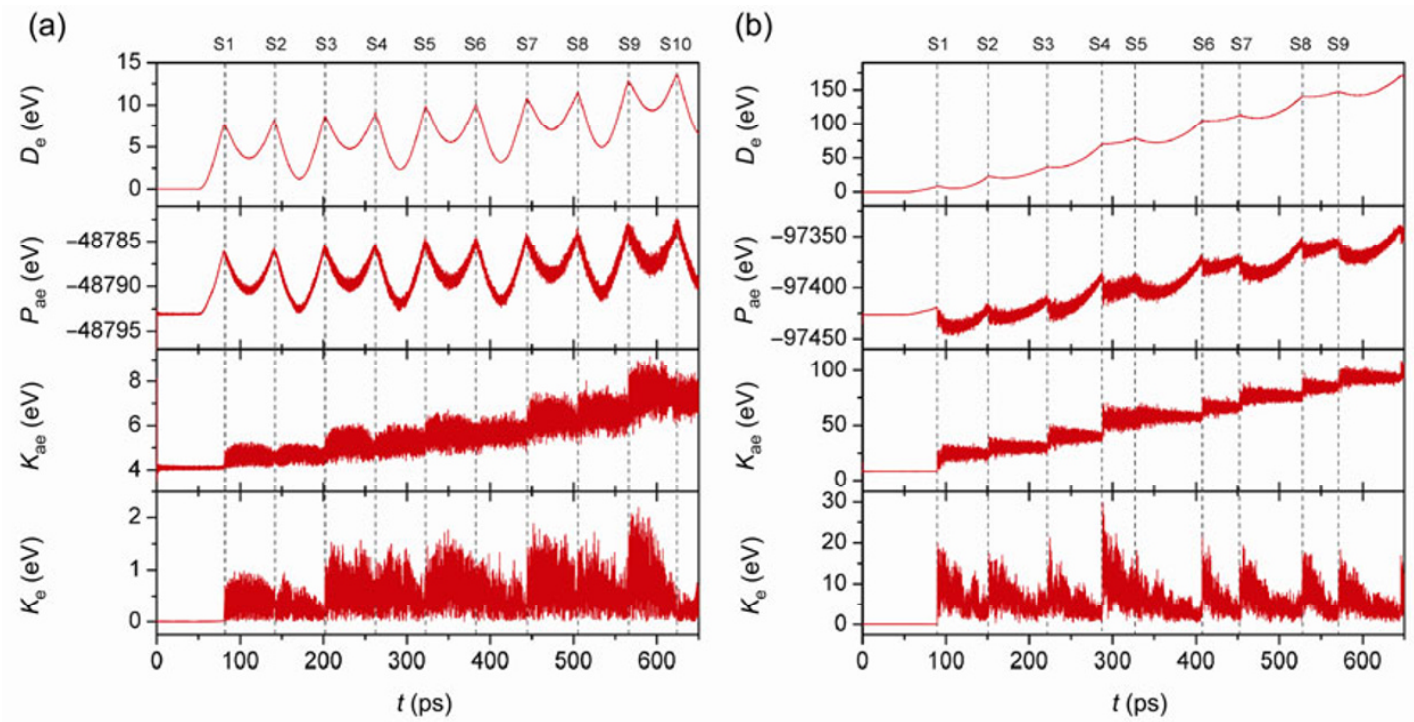

Fig. 11 Energy components and time evolution in multilayer graphene, (a) three-layer system, (b) five-layer system [53]. 
to potential energy. Since no increase in vibration energy is observed as $K_{\mathrm{e}}$ decreases, we expect that the latter would dominate the transformation.

(4) Slow release of $P_{\text {ae }}$ and reversible energy

A careful examination of Fig. 11 reveals an important feature of the energy dissipation. In the course of slip, the decrease in $P_{\text {ae }}$ consists of two stages, a fast drop in a few picoseconds followed by a slow relaxation that may last for 20-30 ps. After $P_{\text {ae }}$ reaches the minimum, it increases again, preparing for a new stick event. As a result, the potential energy exhibits a U-type variation in the time interval between the slip and the next stick, which can be seen more clearly in the 3-layer system. The slow decrease in stored energy was also reported by Lei and Leng [44] in their MD simulations and was attributed to material relaxation. While it is true that material relaxations do release energy in such a manner, we found that a cyclic energy exchange between graphene and the external driver is responsible for the slow release and the U-type variation of $P_{\mathrm{ae}}$. More specifically, a part of the potential energy is fed back to the driver, leading to a decrease in external work $D_{\mathrm{e}}$, as can be seen in Fig. 11, indicating that the external driver receives a part of the energy from the graphene system. The exchange constitutes a conservative part of energy that circulates reversibly in a frictional system. The larger this reversible energy, the less is the energy dissipation. There would be no dissipation at all if the energy is fully reversible. This is a frictionless state predicted by the Tomlinson model when stick-slip disappears.

It is interesting to compare the modes of dissipation discussed in this section with those in stick-slip friction between surfaces separated by confined liquids, as examined by Klein [54]. It was demonstrated in [54] that the major part of the stored energy is dissipated as viscous heating of the confined film during slip while the rest is dissipated as mechanical oscillations due to momentum transfer to the external system. This is consistent with what we see in Fig. 11, where there is a sudden increase in atomic thermal energy $\left(K_{\mathrm{ae}}\right)$ at the time of slip, accompanied by significant oscillations of the graphene layers $\left(K_{\mathrm{e}}\right)$. However, we note that the slip-induced fast dissipation is followed by a relatively slow release of potential energy prior to the onset of the next stick, which was not observed in [54]. The discrepancy may result from the difference in system setup in Klein's case, where the top surface is connected to a spring whose end is pulled at a constant velocity. In our simulation, the top graphene layer is directly pulled by the driver so the elastic energy is stored mostly in the form of atomic deformation within the graphene layer. As a result, a part of the internal energy will be released in a relatively slow process of relaxation. The most significant aspect of this comparison is that in addition to the momentum transfer reported in [54], we show that there is an exchange of energy between the internal and external systems and the amount of this reversible energy has a crucial impact on atomicscale friction.

Here, we have shown that the potential energy stored in the stick phase may not be completely dissipated, raising the question as to how to determine the coefficient of dissipation, i.e., the irreversibly dissipated energy divided by the total stored energy. In fact, the concept of the dissipation coefficient was introduced in the cobblestone model (see Eq. (6) in Section 4), but little work has been undertaken to clarify it. It is simply assumed in most studies that all the stored energy has been dissipated. This leads us to emphasize that it is crucial in future studies of friction to clearly define the coefficient of energy dissipation.

\section{Concluding remarks}

There is a prevalent mystery in the fundamental study of friction between two solid surfaces sliding past each other. How is frictional work dissipated, especially when material damage and wear are not involved? To answer this question, the mechanisms of energy dissipation have been discussed in this paper by reviewing the models and research that apply to atomic-scale friction, leading to the following concluding remarks.

(1) According to the Tomlinson model, energy dissipation in atomic-scale friction initiates from the mechanical instability that induces stick-slip motions and atomic vibrations. The occurrence of the stick-slip motion can be predicted by a criterion that involves 
two features of the system, namely, the corrugation of surface energy and the stiffness of surface bonds.

(2) The models lead to a logical conclusion that the magnitude of friction and energy dissipation would be greatly affected by the structural commensurability between two surfaces in contact, and a frictionless state (superlubricity) can be achieved in a perfectly incommensurate system. The prediction has been confirmed by a few carefully designed experiments, such as those using QCM and AFM, but it does not match the behavior of macroscopic friction encountered in engineering.

(3) The cobblestone model suggests a slightly different picture of energy dissipation: Sliding consists of a series of events of surface separation and approach, and it is the energy loss in the cycle of surface separation-approach that determines the magnitude of friction. In this way, the mechanism of energy dissipation has been related to adhesion hysteresis, which may be appropriate for boundary lubrication. The model provides an estimate for the interfacial shear strength on the basis of the energy loss in adhesion hysteresis.

(4) In the phononic description, the sudden release of stored energy corresponds to the excitation of excess phonons that will decay consequently to a new state of equilibrium by internal and interfacial damping. As a consequence, it is theoretically possible to achieve a nearly frictionless state for an isolated system of sufficiently small size because phonons will remain at isolated discrete modes with no interaction, implying no energy dissipation occurs.

(5) In friction systems involving metallic materials, electronic damping may play an important role. Several carefully designed experiments reveal that there is a considerable drop in frictional force when the temperature goes below the superconductivity transition temperature, illustrating a dominance of electronic friction, but other experiments do not confirm the observations. The contribution from electronic damping remains an open question.

(6) In addition to atomic vibration, other mechanisms also contribute to energy dissipation. Molecule activation and relaxation, for example, are particularly important to the frictional dissipation of viscoelastic materials. In most engineering applications, friction is thought to be accompanied by wear. However, the study of wearless friction should be emphasized because the majority of frictional work is converted into heat instead of wear debris, and wearless dissipation is more difficult to understand and resolving the mystery is a challenging mission.

(7) MD simulations for multilayer graphene show that there is a portion of potential energy circulating reversibly between the system and the external driver. In other words, the dissipated component takes only a portion of the total stored energy. This creates a possibility of achieving a nearly frictionless state by minimizing energy dissipation.

\section{Acknowledgement}

The authors would like to express their appreciations to the financial support from the National Natural Science Foundation of China under the grant Nos. of 51075526, 51005129, and 51021064. The authors would like to thank Enago (www.enago.cn) for the English language review.

Open Access: This article is distributed under the terms of the Creative Commons Attribution Noncommercial License which permits any noncommercial use, distribution, and reproduction in any medium, provided the original author(s) and source are credited.

\section{References}

[1] Dowson D. History of Tribology. London (UK): Professional Engineering Publishing Limited, 1998.

[2] Bowden F P, Tabor D. Friction and Lubrication of Solids. Oxford (UK): Oxford University Press, 1954.

[3] McClelland G M. Friction between weekly interacting smooth surfaces. In Adhesion and Friction. Kreuzev and Grinze, Ed. Berlin: Series in Surface Science, Springer Verlag, 1989: 1-16.

[4] Tabor D. Friction as a dissipative process. In Fundamentals of Friction: Macroscopic and Microscopic Processes. Singer I L and Pollock H M, Ed. Dordrecht: Kluwer, 1992: 3-20.

[5] Tomlinson G A. A molecular theory of friction. Phil Mag 7: 905-939 (1929)

[6] Klein J, Kumacheva E, Mahalu D, Perahla D, Fetters L J. Reduction of frictional forces between solid surfaces bearing polymer brushes. Nature 370: 634-636 (1994)

[7] Raviv U, Klein J. Fluidity of bound hydration layers. Science 297: 1540-1543 (2002) 
[8] Klein J. Hydration lubrication. Friction 1: in press (2013)

[9] Prandtl L, Gedankenmodell E, zur kinetischen Theorie der festen Körper. ZAMM 8: 85-106 (1928)

[10] Tomanek D, Zhong W, Thomas H. Calculation of an atomically modulated friction force in atomic-force microscopy. Europhys Lett 15: 887-892 (1991)

[11] Gnecco E, Bennewitz R, Gyalog T, Meyer E. Friction experiments on the nanometer scale. Journal of Physics: Condensed Matter 13: R619-642 (2001)

[12] Gnecco E, Bennewitz R, Gyalog T, Loppacher, Ch, Bammerlin M, Mayer E, Guntherodt H J. Velocity dependence of atomic friction. Physical Review Letters 84(6): 1172-1175 (2000)

[13] Sang Y, Dube M, Grant M. Thermal effects on atomic friction. Physical Review Letters 87(17): 174301 (2001)

[14] Suda H. Origin of friction derived from rupture dynamics. Langmuir 17: 6045-6067 (2001)

[15] Xu Z M, Huang P. Composite oscillator model for the energy dissipation mechanism of friction. ACTA PHYSICA SINICA 55(5): 2427-2432 (2006)

[16] Frenkel J, Kontorova T. On the theory of plastic deformation and twinning. J Phys USSR 1: 137 (1939)

[17] Aubry S. Critical behavior at the transition by breaking of analyticity in the discrete Frenkel-Kontorova model. $J$ Phys C 16: 1593-1608 (1983)

[18] Floria L M, Mazo J J. Dissipative dynamics of the FrenkelKontorova model. Adv Phys 45(6): 505-598 (1996)

[19] Braun O M, Dauxois T, Paliy M V, Peyrard M. Nonlinear mobility of the generalized Frenkel-Kontorova model. Phys Rev E 55(3): 3598-3612 (1997)

[20] Strunz T, Elmer F J. On the sliding dynamics of the Frenkel-Kontorova model. In Physics of Sliding Friction. B N J Persson B N J and Tosatti E, Ed. Dordrecht: Kluwer, 1996: 149-162.

[21] Weiss $M$ and Elmer, F J. Dry friction in the FrenkelKontorova-Tomlinson model: Static properties. Phys Rev B 53(11): 7539-7549 (1996)

[22] Weiss $\mathrm{M}$ and Elmer, F J. Dry friction in the FrenkelKontorova-Tomlinson model: dynamical properties. Zeitschrift Fur Physik B 104: 55-69 1(997)

[23] Hu Y Z, Zhang T, Ma T B, Wang H. Molecular dynamics simulations on atomic friction between self-assembled monolayers: Commensurate and incommensurate sliding. Computational Material Science 38(1): 98-104 (2006)

[24] Krim J, Solina D H, Chiarello R. Nanotribology of a Kr monolayer: a quatz crystal microbalance study of atomicscale friction. Physical Review Letters 66: 181-184 (1991)

[25] Cieplak M, Smith E D, Robbins M O. Molecular origins of friction. Science 265: 1209-1212 (1994)
[26] Dienwiebel M, Verhoeven G S, Pradeep N, Frenken J W, Heimberg J A, Zandbergen H W. Superlubricity of graphite. Physical Review Letters 92(12): 126101 (2004)

[27] Tabor D. The role of surface and intermolecular forces in thin film lubrication. In Microscopic Aspects of Adhesion and Lubrication. Georges J M, Ed. Amsterdam: Elsevier, 1980: 651-679.

[28] Homola A M, Israilachvili J N, Gee M L, McGuiggan P M. Measurements of and relation between the adhesion and friction of two surfaces separated by molecularly thin liquidfilms. Journal of Tribology, Trans ASME 111(4): 675-682 (1989)

[29] Berman A D, Israelachivili J N. Microtribology and Microrheology of Molecularly Thin Liquid Films. In Modern Tribology Handbook. Bhushan B, Ed. Boca Raton: CRC Press, 2001: 568-610.

[30] Chen Y L, Helm C, Israelachivili J N. Molecular mechanisms associated with adhesion and contact angle hysteresis of monolayer surfaces. Journal of Physical Chemistry 95: 10736-10747 (1991)

[31] Buldum A, Leitner D M, Ciraci S. Model of phononic energy dissipation in friction. Physical Review B 59(24): 16042-16046 (1999)

[32] Sokoloff J B. Possible nearly frictionless sliding for mesoscopic solids. Physical Review Letters 71(21): 3450 3453 (1993)

[33] Kajita S, Washizu H, Ohmori T. Deep bulk atoms in a solid cause friction. Epl-Europhys Lett 87(6): 66002 (2009)

[34] Persson B N J, Nitzan A. Linear sliding friction: On the origin of the microscopic friction for Xe on silver. Surface Science 367(3): 261-275 (1996)

[35] Dayo A, Alnasrallah W, Krim J. Superconductivitydependent sliding friction, Physical Review Letters 80: 1690-1693 (1998)

[36] Persson B N J. Applications of surface resistivity to atomic scale friction, to the migration of hot adatoms, and to electrochemistry. Journal of Chemical Physics 98: 16591672 (1993)

[37] Fois G, Bruschi L, d'Apolito L, Mistura G, Torre B, de Mongeot F B, Boragno C, Buzio R, Valbusa U. Lowtemperature static friction of $\mathrm{N}_{2}$ monolayers on $\mathrm{Pb}(111)$. Journal of Physical: Condensed Matter 19: 305013 (2007)

[38] Kisiel M, Gnecco E, Gysin U, Marot L, Rast S, Meher E. Suppression of electronic friction on $\mathrm{Nb}$ films in the superconducting state. Nature Materials 10: 119-122 (2011).

[39] Acay A. Acoustics of friction. J Aoust Soc Am 111(4): 1525-1548 (2002) 
[40] Bruesch P. Phonons: Theory and Experiments I, Lattice dynamics and models of interatomic forces. Berlin: pringerVerlag, 1982.

[41] Johansen A, Sornette D. Acoustic radiation controls dynamic friction: evidence from a spring-block experiment. Physical Review Letters 82(25): 5152-5155 (1999)

[42] Lifshitz R, Roukes M L. Thermoelastic damping in microand nanomechanical systems. Physical Review B 61(8): 5600-5609 (2000)

[43] Bureau L, Caroli C, Baumberger T. Elasticity and onset of frictional dissipation at a non-sliding and multi-contact interface. Proc R Soc Lond A 459: 2787-2805 (2003)

[44] Lei Y J, Leng Y S. Stick-slip friction and energy dissipation in boundary lubrication. Physical Review Letters 107: 147801 (2011)

[45] Knorr D B, Widjaja P, Acton O, Overney R M. Molecular friction and mode coupling in organic monolayers and polymer films. Journal of Chemical Physics 134: 104502 (2011)

[46] Scott S, Gray T, Overnet R M. Molecule dissipation

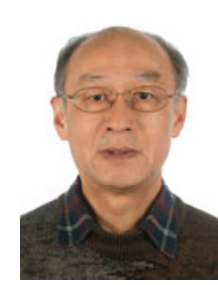

Yuan-zhong HU graduated in 1968 from Tsinghua University, and received his PhD in 1985. Since then he joined the SKLT, Tsinghua University, as an associate professor and later a professor. During 1989-1992, he worked in Norwegian Technical University, Norway, and in Northwestern University, US, as a postdoctoral fellow. He stayed in the US again during 1997.5-1998.12, as a visiting scholar. His research interests include EHL, phenomena of nanoscopic friction in the heterogenerous relaxation regime of a glass former. Journal of Chemical Physics 123: 134902 (2005)

[47] Amiri M, Khonasri M M. On the thermodynamics of friction and wear - a review. Entropy 12: 1021-1049 (2010)

[48] Huq M Z, Celis, J P. Expressing wear rate in sliding contacts based on dissipated energy. Wear 252: 375-383 (2002)

[49] Uetz H, Fohl J. Wear as an energy transformation process. Wear 49: 253-264 (1978)

[50] Fleisher G. Energetische methode der bestimmung des verschleibes. Schmierungstechnik 4: 9-22 (1973)

[51] Wen S Z, Huang P. Principle of Tribology. Beijing: Tsinghua University Press, 2008.

[52] Gotsmann B, Lantz M A. Atomistic wear in single asperity sliding contact. Physical Review Letters 101: 125501 (2008)

[53] $\mathrm{Xu} \mathrm{L.} \mathrm{Research} \mathrm{on} \mathrm{the} \mathrm{friction} \mathrm{and} \mathrm{energy} \mathrm{dissipation}$ behavior of few-layer graphene. Ph.D. Thesis. Beijing (China): Tsinghua University, 2012.

[54] Klein J. Frictional dissipation in stick-slip sliding. Physical Review Letters 98: 056101 (2007)

Mixed lubrication, Wear dynamics, Nano-Tribology, Thin film rheology, Molecular dynamics simulations, etc., and in these areas he has more than 100 papers published on international journals. Prof. Hu received the Edmond E. Bisson Award from STLE in 2003 due to his contributions to the numerical analysis of mixed lubrication, and he is now an Associate Editor of the Proceedings of IMechE, Part J, Journal of Engineering Tribology. 